\title{
On Teleparallel Quantum Gravity in Schwarzschild Space-Time
}

\author{
S. C. Ulhoa ${ }^{1,2}$ and R. G. G. Amorim ${ }^{1,2}$ \\ ${ }^{1}$ Instituto de Física, Universidade de Brasília, 70910-900 Brasília, DF, Brazil \\ ${ }^{2}$ Faculdade Gama, Universidade de Brasília, Setor Leste (Gama), 72444-240 Brasília, DF, Brazil \\ Correspondence should be addressed to S. C. Ulhoa; sc.ulhoa@gmail.com
}

Received 12 March 2014; Revised 1 May 2014; Accepted 2 May 2014; Published 21 May 2014

Academic Editor: Elias C. Vagenas

Copyright (C) 2014 S. C. Ulhoa and R. G. G. Amorim. This is an open access article distributed under the Creative Commons Attribution License, which permits unrestricted use, distribution, and reproduction in any medium, provided the original work is properly cited. The publication of this article was funded by SCOAP ${ }^{3}$.

We present the quantization process for Schwarzschild space-time in the context of Teleparallel gravity. In order to achieve such a goal we use the Weyl formalism that establishes a well-defined correspondence between classical quantities which are realized by functions and quantum ones which are realized by operators. In the process of quantization we introduce a fundamental constant that is used to construct what we call the quantum of matter by the imposition of periodic conditions over the eigenfunction.

\section{Introduction}

The dynamical behavior of physical systems can be realized essentially by two descriptions of reality, the classical approach to which the physical world evolves deterministically and the quantum one which describes nature by means of the concept of probabilities. The quantum description is usually obtained from classical description through appropriate processes, the so-called quantization procedures [1-3]. The first ideas about quantization emerged in 1925, with Heisenberg who proposed a description of quantum mechanics based solely on terms of observable quantities [4]. Thus Heisenberg used an amplitude multiplication rule that later Born has identified to a matrix calculation [5]. In such a formalism scope, important results have been yielded, among them we point out the quantization of the harmonic oscillator solution and the achievement of a commutation relation between position and its conjugated momentum. Born included Jordan in such a discussion and together they have generalized what was known so far for systems with arbitrary degrees of freedom, they have introduced the canonical transformations for this context as well [5]. In 1926, Pauli gave his contribution to the development of the quantization procedure by showing how to obtain the hydrogen spectrum from this formalism [6]. Dirac, independently, was able to establish the connection between classical and quantum mechanics, relying on the Hamilton-Jacobi formulation of classical mechanics and using an algebraic formalism [6, 7]. Born and Wiener have focused on matrix approach which has led to the representation of the Hamiltonian (until then a classical function) in terms of operators; in this sense, arose the first quantization procedure as discussed in $[4,7]$ and in the references therein.

Since then, the process of quantizing a physical system has become a controversial subject and several methods have been proposed; among them stand out the canonical quantization, path integral quantization, and Weyl quantization which will be focused in this paper. The first two methods are based on Dirac rules and Feynman generating functional, respectively [8]. Both have some problems such as the noninvariance under canonical transformations and they seem to be not extendable to noneuclidian phase spaces. Particularly the canonical quantization method leads to difficulties in the understanding of the quantum-classical limit. On the other hand, the Weyl quantization procedure, developed in 1927 [9], is a more robust approach; in such a method there is a well-defined mathematical operation with a clear correspondence rule between classical functions and quantum operators. Even though these methods are largely used to quantize a classical field, such processes are far from being unanimously accepted; for instance the arising operators order is very controversial $[8,10]$.

We would like to implement the Weyl quantization procedure to construct a quantum theory of gravitation. So 
far every attempt to address the problem of quantum gravity is based on General Relativity which is the most receptive theory of gravitation in the scientific community. Such an approach is revealed to be ill defined; for instance we point out the problem of time in loop quantum gravity [11] and the nonrenormalization problem $[12,13]$. In our opinion those problems arise from the fact that General Relativity is not a self-consistent theory since it presents some difficulties that has not been overcome over the years such as the problem of gravitational energy [14-16]. Therefore we will work with an alternative theory of gravitation, the so-called Teleparallelism Equivalent to General Relativity. The reason for such a choice is very simple indeed: Teleparallel gravity allows the existence of a gravitational energy-momentum vector. Such a feature is not present in General Relativity, although both theories are equivalent when it comes to the dynamics of the gravitational field. The geometry in which Teleparallel gravity is constructed is richer than the Riemannian geometry; this yields a wider point of view for Teleparallelism in the analysis of what is going on in the space-time, mainly in the definition of conserved quantities. Teleparallel gravity has been developed and tested over the years in what concerns its classical features [17-23] and in our opinion it seems to be a plausible theory of gravitation. However there are few attempts to quantize this theory; for instance we refer the set of papers [24-27] which were developed as an application of Dirac's method to TEGR. Hence we intent to give our contribution in this process by analyzing the quantum version of Schwarzschild's solution of field equations that arises from the identification $\mathscr{H}=e t^{(0) 0}$ in Weyl's prescription.

The paper is organized as follows. In Section 2, the Weyl quantization procedure is detailed and some basic ideas of Teleparallel gravity are presented. In Section 3, we develop our version of a quantum theory of gravitation for Schwarzschild's solution. Thus we introduce a new fundamental constant, necessary for the quantization procedure, which leads to the definition of a quantum of matter. Finally we present our concluding remarks.

Notation. Space-time indices $\mu, v, \ldots$ and $\mathrm{SO}(3,1)$ indices $a, b, \ldots$ run from 0 to 3 . Time and space indices are indicated according to $\mu=0, i, a=(0)$, (i). The tetrad field is denoted by $e_{\mu}^{a}$ and the determinant of the tetrad field is represented by $e=\operatorname{det}\left(e_{\mu}^{a}\right)$. The tetrad field is related to the metric by $e_{\mu}^{a} e_{a \nu}=g_{\mu \nu}$. In addition we adopt units, where $G=c=1$, unless otherwise stated.

\section{Theoretical Framework}

2.1. Weyl Quantization. In this subsection, we present a quantization procedure called Weyl quantization. We would like to remark that Weyl quantization, in opposition to canonical procedure, is a well-defined mathematical framework and can be extended to study of noneuclidian phase spaces. Furthermore, using Weyl procedure, we can observe easily the correspondence principle. In this sense, we consider a classical system described by $n$ variables which will be denoted by $z_{1}, z_{2}, \ldots, z_{n}$; those variables would be quantized by the prescription:

$$
\left(z_{1}, z_{2}, \ldots, z_{n}\right) \longrightarrow\left(\widehat{z}_{1}, \widehat{z}_{2}, \ldots, \widehat{z}_{n}\right) .
$$

When the classical variables $z_{1}, z_{2}, \ldots, z_{n}$ are quantized by the above rule, the functions $f$ defined on those variables are immediately quantized. This quantization of the functions $f$ occurs by Weyl's map, $\mathscr{W}: f \rightarrow \widehat{f}=\mathscr{W}[f]$, which is given by

$$
\begin{aligned}
& \mathscr{W}[f]\left(z_{1}, z_{2}, \ldots, z_{n}\right) \\
& :=\frac{1}{(2 \pi)^{n}} \int d^{n} k d^{n} z f\left(z_{1}, z_{2}, \ldots, z_{n}\right) \exp \left(i \sum_{l=1}^{n} k_{l}\left(\widehat{z}_{l}-z_{l}\right)\right) .
\end{aligned}
$$

This quantum-classical correspondence is called Weyl quantization. Under the formal mathematical viewpoint, the Weyl method is used to formulate the Groenewold-Moyal quantum mechanics [28]. The kernel of this transformation is given by

$$
\Delta(\widehat{z}, z)=\frac{1}{(2 \pi)^{n}} \int d^{n} k \exp \left(i \sum_{l=1}^{n} k_{l}\left(\widehat{z}_{l}-z_{l}\right)\right) .
$$

In this way, the Weyl map is written by

$$
\mathscr{W}[f]\left(z_{1}, z_{2}, \ldots, z_{n}\right):=\int d^{n} z \Delta(\widehat{z}, z) f\left(z_{1}, z_{2}, \ldots, z_{n}\right) .
$$

Formally the set of operators $\widehat{z}_{i}$ form a noncommutative space [9]. The construction of this noncommutative space is given by the replacement of local coordinates $z_{i}$ by the Hermitian operators $\widehat{z}_{i}$, which leads to the following commutation relation:

$$
\left[\widehat{z}_{i}, \widehat{z}_{j}\right]=i \alpha_{i j}
$$

where $\widehat{z}_{i}$ are operators of a noncommutative algebra and $\alpha_{i j}$ is an antisymmetric tensor. Thus, the product of two operators in noncommutative space is given by $\mathscr{W}[f(z)] \mathscr{W}[g(z)]=$ $\mathscr{W}[f(z) \star f(z)]$, where the Moyal (or star) product is defined by

$$
f(z) \star g(z)=f(z) \exp \left[\frac{i}{2} \alpha^{i j} \overleftarrow{\partial}_{i} \vec{\partial}_{j}\right] g(z)
$$

As an example let us consider the function $f\left(z_{1}, z_{2}\right)=z_{1}^{2}+$ $2 z_{1} z_{2}+z_{2}^{2}$. Applying the Weyl procedure in this function, we obtain

$$
\widehat{f}\left(\widehat{z}_{1}, \widehat{z}_{2}\right)=\widehat{z}_{1}^{2}+\widehat{z}_{1} \widehat{z}_{2}+\widehat{z}_{2} \widehat{z}_{1}+\widehat{z}_{2}^{2}
$$

We realized that the Weyl quantization eliminates the ambiguity in variables ordering present in canonical procedure. Another advantage in Weyl procedure is its use in the quantization of nonpolynomial functions [29]. 
2.2. Teleparallel Equivalent to General Relativity (TEGR). In this subsection we briefly recall the ideas concerning Teleparallel gravity which can be tracked back to the 1930s when Einstein made an attempt to unify gravitation and electromagnetism [30]. In this theory the dynamics of the field relies on the tetrads, $e_{\mu}^{a}$, rather than on the metric tensor, $g_{\mu \nu}$. It can be formally described by means of a Weitzenböck geometry [31], in which the Cartan connection, $\Gamma_{\mu \lambda \nu}=$ $e_{\mu}^{a} \partial_{\lambda} e_{a v}$, plays a central role. Thus the torsion associated with such a connection is given by

$$
T_{\lambda \nu}^{\mu}=e_{a}^{\mu}\left(\partial_{\lambda} e_{\nu}^{a}-\partial_{\nu} e_{\lambda}^{a}\right)
$$

or simply $T_{\lambda v}^{\mu}=e_{a}^{\mu} T_{\lambda \nu}^{a}$, where $T_{\lambda v}^{a}=\partial_{\lambda} e_{\nu}^{a}-\partial_{\nu} e_{\lambda}^{a}$.

We intend to show the equivalence between General Relativity and Teleparallel gravity by showing the equivalence between the geometrical frameworks of both theories. Firstly we note that the Christoffel symbols $\left({ }^{0} \Gamma_{\mu \lambda \nu}\right)$ yield a vanishing torsion tensor due to its symmetric features. The Cartan connection and the Christoffel symbols are related by the following mathematical identity:

$$
\Gamma_{\mu \lambda \nu}={ }^{0} \Gamma_{\mu \lambda \nu}+K_{\mu \lambda \nu}
$$

where

$$
K_{\mu \lambda \nu}=\frac{1}{2}\left(T_{\lambda \mu \nu}+T_{\nu \lambda \mu}+T_{\mu \lambda \nu}\right)
$$

is the contortion tensor. In the same way the Cartan connection yields a vanishing scalar curvature. Thus in the Weitzenböck geometry there is a vanishing curvature while in the Riemann geometry there is a vanishing torsion. Both geometries are related by expression (9), from which it is possible to obtain the relation

$$
e R(e) \equiv-e\left(\frac{1}{4} T^{a b c} T_{a b c}+\frac{1}{2} T^{a b c} T_{b a c}-T^{a} T_{a}\right)+2 \partial_{\mu}\left(e T^{\mu}\right),
$$

where $e$ is the determinant of the tetrad field, $T_{a}=T_{b a}^{b}$, and $R(e)$ is the scalar curvature constructed out in terms of such a field. Therefore we choose the Lagrangian density, in the realm of Teleparallel gravity, as

$$
\mathfrak{L}=-k e\left(\frac{1}{4} T^{a b c} T_{a b c}+\frac{1}{2} T^{a b c} T_{b a c}-T^{a} T_{a}\right)-\mathfrak{Q}_{M},
$$

where $k=1 / 16 \pi$ and $\mathfrak{\Omega}_{M}$ stands for the Lagrangian density for the matter fields. It is worthy to mention that the total divergence had been dropped out in the construction of the Lagrangian density, since it does not contribute to the field equations. It also should be noted, from (11), that the geometrical part of this Lagrangian density is exactly the HilbertEinstein Lagrangian density. Hence both theories share the same dynamical properties. However in Teleparallel gravity it is possible to define a gravitational energy-momentum tensor. Let us rewrite the Lagrangian density as

$$
\mathfrak{Q} \equiv-k e \Sigma^{a b c} T_{a b c}-\mathfrak{Q}_{M},
$$

where

$$
\Sigma^{a b c}=\frac{1}{4}\left(T^{a b c}+T^{b a c}-T^{c a b}\right)+\frac{1}{2}\left(\eta^{a c} T^{b}-\eta^{a b} T^{c}\right) .
$$

Then the field equations can be derived from (13) using a variational derivative with respect to $e^{a \mu}$; they read

$$
e_{a \lambda} e_{b \mu} \partial_{\nu}\left(e \Sigma^{b \lambda v}\right)-e\left(\Sigma_{a}^{b v} T_{b \nu \mu}-\frac{1}{4} e_{a \mu} T_{b c d} \Sigma^{b c d}\right)=\frac{1}{4 k} e T_{a \mu},
$$

where $\delta \mathfrak{Q}_{M} / \delta e^{a \mu}=e T_{a \mu}$. Those equations may be rewritten as

$$
\partial_{\nu}\left(e \Sigma^{a \lambda \nu}\right)=\frac{1}{4 k} e e_{\mu}^{a}\left(t^{\lambda \mu}+T^{\lambda \mu}\right)
$$

where $T^{\lambda \mu}=e_{a}^{\lambda} T^{a \mu}$ and

$$
t^{\lambda \mu}=k\left(4 \Sigma^{b c \lambda} T_{b c}^{\mu}-g^{\lambda \mu} \Sigma^{b c d} T_{b c d}\right) .
$$

In view of the antisymmetry property $\Sigma^{a \mu \nu}=-\Sigma^{a \nu \mu}$, it follows that

$$
\partial_{\lambda}\left[e e_{\mu}^{a}\left(t^{\lambda \mu}+T^{\lambda \mu}\right)\right]=0
$$

which is local balance equation. Therefore such equation leads to the following continuity equation:

$$
\frac{d}{d t} \int_{V} d^{3} x e e_{\mu}^{a}\left(t^{0 \mu}+T^{0 \mu}\right)=-\oint_{S} d S_{j}\left[e e_{\mu}^{a}\left(t^{j \mu}+T^{j \mu}\right)\right]
$$

Thus we identify $t^{\lambda \mu}$ as the gravitational energy-momentum tensor $[32,33]$.

Then, as usual, the total energy-momentum vector is defined by [34]

$$
P^{a}=\int_{V} d^{3} x e e_{\mu}^{a}\left(t^{0 \mu}+T^{0 \mu}\right)
$$

where $V$ is a volume of the three-dimensional space. We point out that the energy-momentum vector is invariant under coordinates transformations and it is sensible to frame transformations as it should be expected.

\section{Quantum Gravity}

In this section we address the problem of quantization of gravity in the framework of Teleparallelism equivalent to General Relativity. Then we start with a stationary space-time:

$$
d s^{2}=g_{00} d t^{2}+g_{11} d r^{2}+g_{22} d \theta^{2}+g_{33} d \phi^{2},
$$

and we bring attention to the fact that this line element is written in spherical coordinates and the metric tensor components are functions of $r$ and $\phi$, solely. In addition we point out that $g_{00}<0$; thus the metric tensor has the proper limit as Minkowski space-time.

There are an infinite number of possible tetrads satisfying the relation $g_{\mu \nu}=e_{\mu}^{a} e_{a v}$ for (21). To fix it we interpret 
the tetrad field as being a reference frame adapted to an observer in space-time. Thus we choose

$$
e_{\mu}^{a}=\left(\begin{array}{cccc}
\sqrt{-g_{00}} & 0 & 0 & 0 \\
0 & \sqrt{g_{11}} \sin \theta \cos \phi & \sqrt{g_{22}} \cos \theta \cos \phi & -\sqrt{g_{33}} \sin \phi \\
0 & \sqrt{g_{11}} \sin \theta \sin \phi & \sqrt{g_{22}} \cos \theta \sin \phi & \sqrt{g_{33}} \cos \phi \\
0 & \sqrt{g_{11}} \cos \theta & -\sqrt{g_{22}} \sin \theta & 0
\end{array}\right),
$$

which is adapted to a stationary observer [35]. In order to obtain the gravitational energy, firstly, we need to obtain the $\Sigma^{(0) 0 i}$ components; they read

$$
\begin{aligned}
& 4 e \Sigma^{(0) 01}= 2\left(\sqrt{g_{33}}+\sqrt{g_{22}} \sin \theta\right) \\
&-\frac{1}{\sqrt{g_{11}}}\left[\sqrt{\frac{g_{33}}{g_{22}}}\left(\frac{\partial g_{22}}{\partial r}\right)+\sqrt{\frac{g_{22}}{g_{33}}}\left(\frac{\partial g_{33}}{\partial r}\right)\right], \\
& 4 e \Sigma^{(0) 02}= 2 \sqrt{g_{11}} \cos \theta \\
&-\frac{1}{\sqrt{g_{22}}}\left[\sqrt{\frac{g_{11}}{g_{33}}}\left(\frac{\partial g_{33}}{\partial \theta}\right)+\sqrt{\frac{g_{33}}{g_{11}}}\left(\frac{\partial g_{11}}{\partial \theta}\right)\right], \\
& e \Sigma^{(0) 03}=0 .
\end{aligned}
$$

We restrict our attention to Schwarzschild space-time to which $g_{00}=(1-2 M / r)=g_{11}^{-1}$, where $M$ is the black hole mass. Thus the only nonvanishing $\Sigma^{(0) 0 i}$ component reads

$$
4 e \Sigma^{(0) 01}=4 r \sin \theta\left[1-\left(1-\frac{2 M}{r}\right)^{1 / 2}\right] .
$$

We recall that $E \equiv P^{(0)}$; then we have

$$
E=4 k \int d^{3} x \partial_{i}\left(e \Sigma^{(0) 01}\right)
$$

which can be represented by $E=\int d^{3} x \mathscr{H}$. Hence $\mathscr{H}=$ $4 k \partial_{i}\left(e \Sigma^{(0) 01}\right)$ which for Schwarzschild space-time yields

$$
\mathscr{H}=4 k \sin \theta\left[1-\frac{(1-M / r)}{(1-2 M / r)^{1 / 2}}\right] .
$$

This is the classical (nonquantum) gravitational Hamiltonian density; it should be noted that it is a tensorial density and as consequence it transforms accordingly under coordinate transformations.

The procedure to quantize this field is formally given by Weyl's prescription which is the following $\theta \rightarrow \widehat{\theta}$ and $r \rightarrow \widehat{r}$, where $\hat{\theta}=i \alpha(\partial / \partial r)$ and $\hat{r}=r$. Here $\alpha$ is a constant with dimension of distance. Thus the commutator between such operators is

$$
[\widehat{\theta}, \widehat{r}]=i \alpha
$$

as defined by relation (8). As a consequence $\mathscr{H} \rightarrow \widehat{\mathscr{H}}$. The constant $\alpha$ is supposed to be very small, since the noncommutativity between $r$ and $\theta$ is not observed in everyday life.
Therefore $\alpha \ll 1$ which leads to $\sin (i \alpha(\partial / \partial r)) \simeq i \alpha(\partial / \partial r)$. After some algebraic manipulations we find that $\widehat{\mathscr{H}}$ is given by

$$
\widehat{\mathscr{H}}=4 k i \alpha\left\{\left[1-\frac{(1-M / r)}{(1-2 M / r)^{1 / 2}}\right] \frac{\partial}{\partial r}+\frac{M / 2 r^{2}}{(1-2 M / r)^{3 / 2}}\right\} .
$$

We immediately see that this operator is antihermitian; therefore it also has real eigenvalues. We suppose an eigenvector/eigenvalue equation as $\widehat{\mathscr{H}} \psi=\epsilon \psi$ which leads to an equation of the form $(\partial \psi / \partial r)+g(r) \psi=0$, where $g(r)$ is written as

$$
g(r)=\left[1-\frac{(1-M / r)}{(1-2 M / r)^{1 / 2}}\right]^{-1}\left[i \frac{\epsilon}{4 k \alpha}+\frac{M / 2 r^{2}}{(1-2 M / r)^{3 / 2}}\right] .
$$

The quantity $\epsilon$ is the eigenvalue. We point out that in our unit system the Hamiltonian density is adimensional; as a consequence the energy has length dimension since it comes from a volume integration of the Hamiltonian density. Therefore the Hamiltonian eigenvalue is adimensional as well and then it is given by $\epsilon=E / M$, where $E$ is the observable of the field.

Since the above equation is a first-order differential equation, its solution is

$$
\psi=\psi_{0} \exp \left(-\int g(r) d r\right)
$$

where $\psi_{0}$ is a constant of integration. It can be chosen to normalize the solution. Let us analyze the consequences of such a solution in the limit $M \ll r$. Then we find

$$
\psi=\psi_{0} \exp \left(-\frac{i \epsilon}{8 k \alpha M} r^{2}\right)
$$

and, in the next step we impose that the solution should assume the same values at the singularity points $r=0$ and $r=2 M$; hence $\psi(0)=\psi(2 M)$. It is well known that $E=M$ for Schwarzschild space-time which leads to the conclusion that $\epsilon=1$. We point out that the value of the gravitational energy yielded by TEGR is the classical observable; thus the eigenvalue of our quantum equation should fulfill such expectation. Therefore we finally have

$$
M=n m_{0},
$$

where $n$ is an integer once $k=1 / 16 \pi$ and $m_{0}=\alpha / 4$. Such a condition arises from the use of the boundary condition $\psi(0)=\psi(2 M)$. In the international unit system we have $m_{0}=\alpha c^{2} / 4 G$ which is the quantum of matter.

\section{Conclusion}

In this paper we have presented a formal procedure to construct a quantum theory of gravity. We have performed our calculations in the realm of Teleparallel gravity due to the arising of a proper energy-momentum vector as one of 
its fundamental features. We have used the Weyl quantization process to obtain operators out of classical quantities; then we establish an eigenvalue/eigenvector equation which reveals the quantum features of the field in the context of Schwarzschild space-time. Such quantum properties are obtained by the imposition of periodic conditions on the eigenfunction which is the solution of $\widehat{\mathscr{H}} \psi=\epsilon \psi$. This leads to the definition of $m_{0}$, the quantum of matter. Thus the black hole mass is quantized in terms of such a parameter. The quantum of matter, in the international unities, is written in terms of gravitational constant, speed of light, and $\alpha$ which is a constant with dimension of length, introduced in the quantization process. Therefore, in order to give the order of magnitude of this new constant, we point out that every piece of matter is formed by electrons as its smallest mass constituents. We recall that other tiny constituents such as quarks are more massive than electrons. Hence we associate the quantum of matter with the electron's mass; this yields $\alpha \sim 10^{-56} \mathrm{~m}$. Bearing this in mind, we think that the electron may have different mechanisms to yield what it is observed, for instance, one responding for matter and another one for charge and spin. We also point out that the results obtained in this paper were derived from a hamiltonian density, $\mathscr{H}=e t^{(0) 0}$, which is not invariant under coordinate transformations. Such a feature is also present when one tries to quantize fields in a curved space-time. In fact we expect a break of the group of diffeomorphism since we substitute coordinates by operators in the construction of a quantum theory of gravitation. This feature would lead to different equations for each coordinate system; however all of them should behave equally in the limit $M / r \ll 1$. Our results may be extended to fundamental particles since their line element can be described by Schwarzschild's solution in isotropic coordinates as obtained in [36].

\section{Conflict of Interests}

The authors declare that there is no conflict of interests regarding the publication of this paper.

\section{References}

[1] F. A. Berezin, "General concept of quantization," Communications in Mathematical Physics, vol. 40, pp. 153-174, 1975.

[2] S. T. Ali and M. Engliš, "Quantization methods: a guide for physicists and analysts," Reviews in Mathematical Physics, vol. 17, no. 4, pp. 391-490, 2005.

[3] D. Giulini, "That strange procedure called quantisation," in Quantum Gravity, vol. 631 of Lecture Notes in Physics, pp. 17-40, Springer, Berlin, Germany, 2003.

[4] P. J. Kennedy and A. P. French, Niels Bohr: A Centenary Volume, Harvard University Press, Cambridge, Mass, USA, 1985.

[5] F. Hund, "Paths to quantum theory historically viewed," Physics Today, vol. 19, no. 8, p. 23, 1966.

[6] J. Hendry, The Creation of Quantum Mechanics and the BohrPauli Dialogue, Reidel, Dordrecht, The Netherlands, 1984.

[7] M. Jammer, The Conceptual Development of Quantum Mechanics, McGraw-Hill, New York, NY, USA, 1966.
[8] S. T. Ali and M. Engliš, "Quantization methods: a guide for physicists and analysts," Reviews in Mathematical Physics, vol. 17, no. 4, pp. 391-490, 2005.

[9] H. Weyl, The Theory of Groups and Quantum Mechanics, Dover, New York, NY, USA, 1931.

[10] J. Shewell, "On the formation of quantum-mechanical operators," American Journal of Physics, vol. 27, p. 16, 1959.

[11] E. Anderson, "The problem of time in quantum gravity," in Classical and Quantum Gravity: Theory, Analysis and Applications, V. R. Frignanni, Ed., chapter 4, Nova, New York, NY, USA, 2012.

[12] A. Shiekh, "Can the equivalence principle survive quantization?" Hyperfine Interactions, vol. 109, p. 105, 1997.

[13] A. Shomer, "A pedagogical explanation for the non-renormalizability of gravity," http://arxiv.org/abs/0709.3555 .

[14] L. D. Landau and E. M. Lifshitz, The Classical Theory of Fields, Butterworth-Heinemann, 4th edition, 1980.

[15] A. Komar, "Covariant conservation laws in general relativity," vol. 113, pp. 934-936, 1959.

[16] J. W. Maluf, "Localization of energy in general relativity," Journal of Mathematical Physics, vol. 36, no. 8, pp. 4242-4247, 1995.

[17] F. W. Hehl, "Four lectures on Poincare Gauge Field Theory," in Cosmology and Gravitation on Spin, Torsion, Rotation and Supergravity, P. G. Bergmann and V. de Sabbata, Eds., NATO Advanced Study Institutes Series, Springer, May 1980.

[18] F. W. Hehl, J. Lemke, and E. W. Mielke, "Two lectures on fermions and gravity," in Geometry and Theoretical Physics, J. Debrus and A. C. Hirshfeld, Eds., pp. 56-140, Springer, Berlin, Germany, 1991.

[19] K. Hayashi and T. Shirafuji, "New general relativity," Physical Review D, vol. 19, no. 12, pp. 3524-3553, 1979.

[20] J. W. Maluf, S. C. Ulhoa, F. F. Faria, and J. F. da Rocha-Neto, “The angular momentum of the gravitational field and the Poincaré group," Classical and Quantum Gravity, vol. 23, no. 22, pp. 62456256, 2006.

[21] J. W. Maluf and S. C. Ulhoa, "On the gravitational angular momentum of rotating sources," General Relativity and Gravitation, vol. 41, no. 6, pp. 1233-1247, 2009.

[22] J. W. Maluf and S. C. Ulhoa, "The energy-momentum of planefronted gravitational waves in the teleparallel equivalent of GR," Physical Review D, vol. 78, no. 4, article 047502, 2008.

[23] S. Ulhoa, "On dark energy and accelerated reference frames," Annalen Der Physik, vol. 524, no. 5, pp. 273-278, 2012.

[24] A. Okołów, "Construction of spaces of kinematic quantum states for field theories via projective techniques," Classical and Quantum Gravity, vol. 30, no. 19, article 195003, 2013.

[25] A. Okołów, "Kinematic quantum states for the Teleparallel Equivalent of General Relativity," General Relativity and Gravitation, vol. 46, article 1653, 2014.

[26] A. Okołów, "Variables suitable for constructing quantum states for the teleparallel equivalent of general relativity I," General Relativity and Gravitation, vol. 46, article 1620, 2014.

[27] A. Okołów, "Variables suitable for constructing quantum states for the teleparallel equivalent of general relativity II," General Relativity and Gravitation, vol. 46, article 1638, 2014.

[28] J. Hancock, M. A. Walton, and B. Wynder, "Quantum mechanics another way," European Journal of Physics, vol. 25, no. 4, pp. 525-534, 2004.

[29] N. Mukunda, G. Marmo, A. Zampini, S. Chaturvedi, and R. Simon, "Wigner-Weyl isomorphism for quantum mechanics on Lie groups," Journal of Mathematical Physics, vol. 46, article 012106, 2005. 
[30] A. Einstein, "Auf die Riemann-Metrik und den Fern-Parallelismus gegründete einheitliche Feldtheorie," Mathematische Annalen, vol. 102, no. 1, pp. 685-697, 1930.

[31] E. Cartan, "On a generalization of the notion of reimann curvature and spaces with torsion," in Cosmology and Gravitation: Spin, Torsion, Rotation, and Supergravity, P. G. Bergmann and V. de Sabbata, Eds., vol. 58 of NATO ASIB Proceedings, pp. 489491, 1980.

[32] V. C. de Andrade, L. C. T. Guillen, and J. G. Pereira, "Gravitational energy-momentum density in teleparallel gravity," Physical Review Letters, vol. 84, no. 20, pp. 4533-4536, 2000.

[33] J. W. Maluf, “The gravitational energy-momentum tensor and the gravitational pressure," Annalen der Physik, vol. 14, pp. 723732, 2005.

[34] J. Maluf, J. da Rocha-Neto, T. Toribio, and K. Castello-Branco, "Energy and angular momentum of the gravitational field in the teleparallel geometry," Physical Review D, vol. 65, article 124001, 2002.

[35] J. W. Maluf, F. F. Faria, and S. C. Ulhoa, "On reference frames in spacetime and gravitational energy in freely falling frames," Classical and Quantum Gravity, vol. 24, no. 10, pp. 2743-2753, 2007.

[36] M. O. Katanaev, "Point massive particle in general relativity," General Relativity and Gravitation, vol. 45, no. 10, pp. 1861-1875, 2013. 

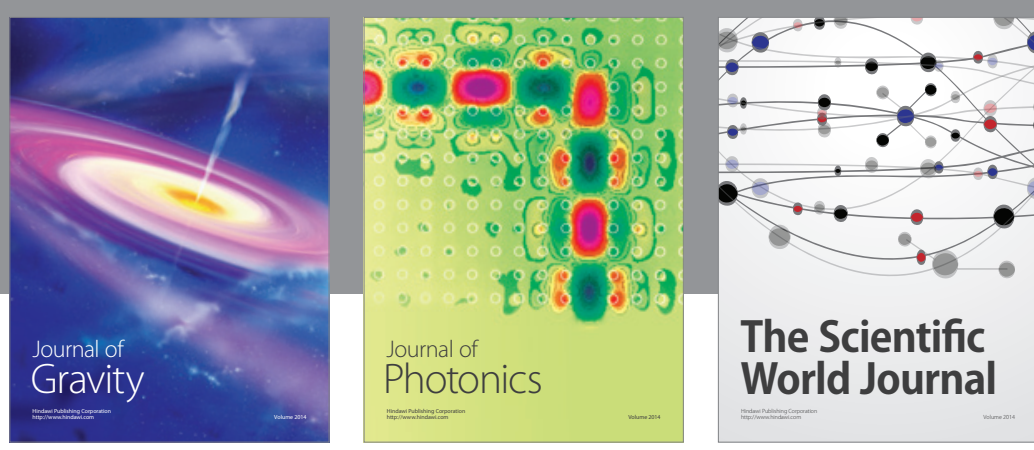

The Scientific World Journal
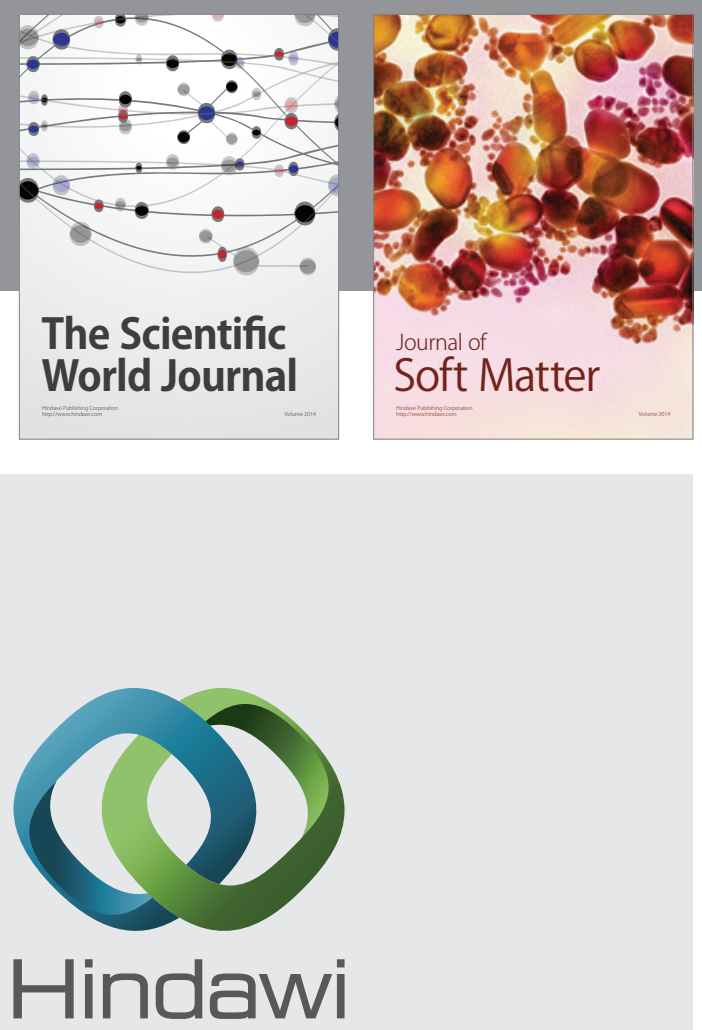

Submit your manuscripts at

http://www.hindawi.com

nternational Journal of

Statistical Mechanics
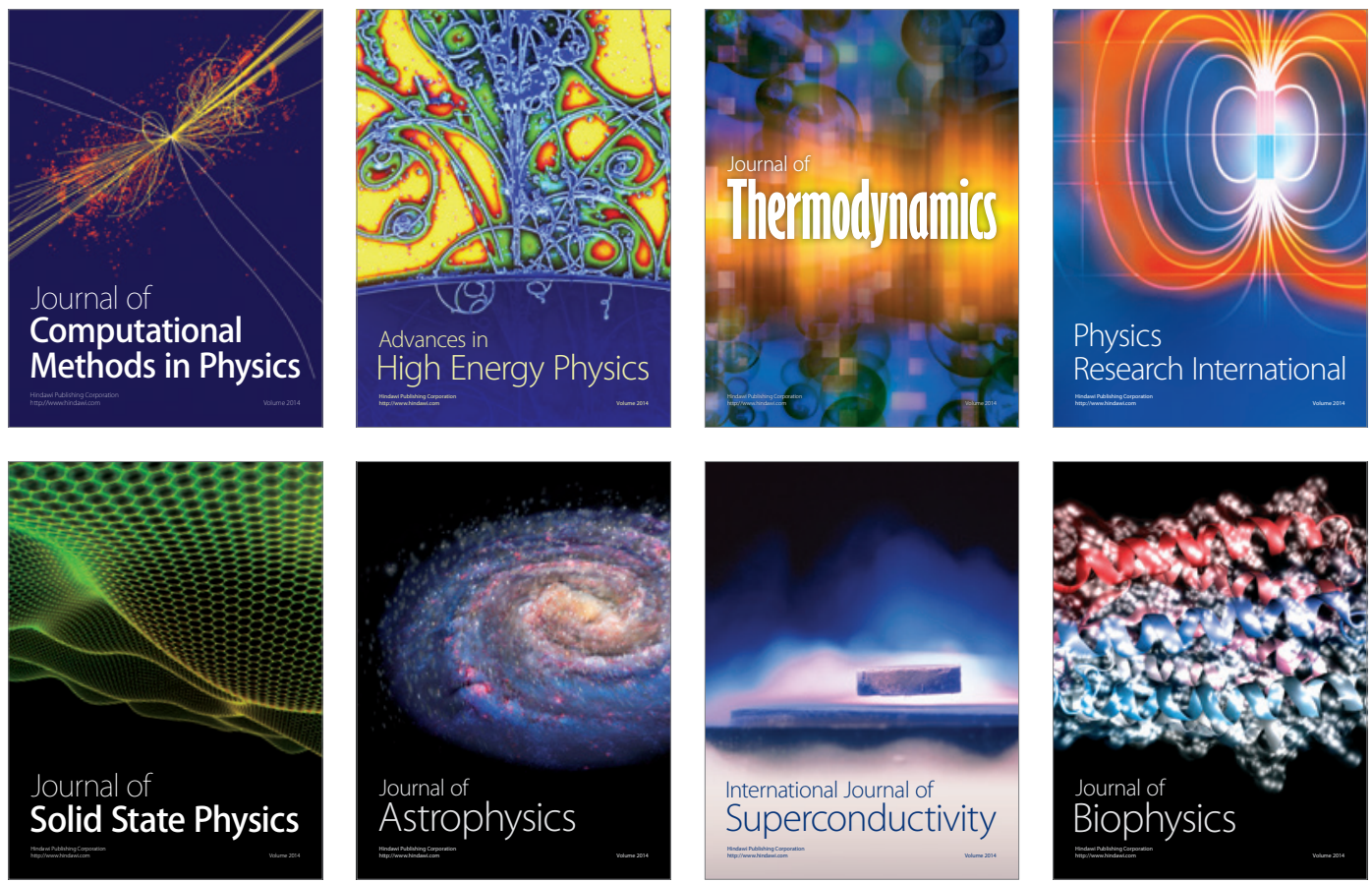
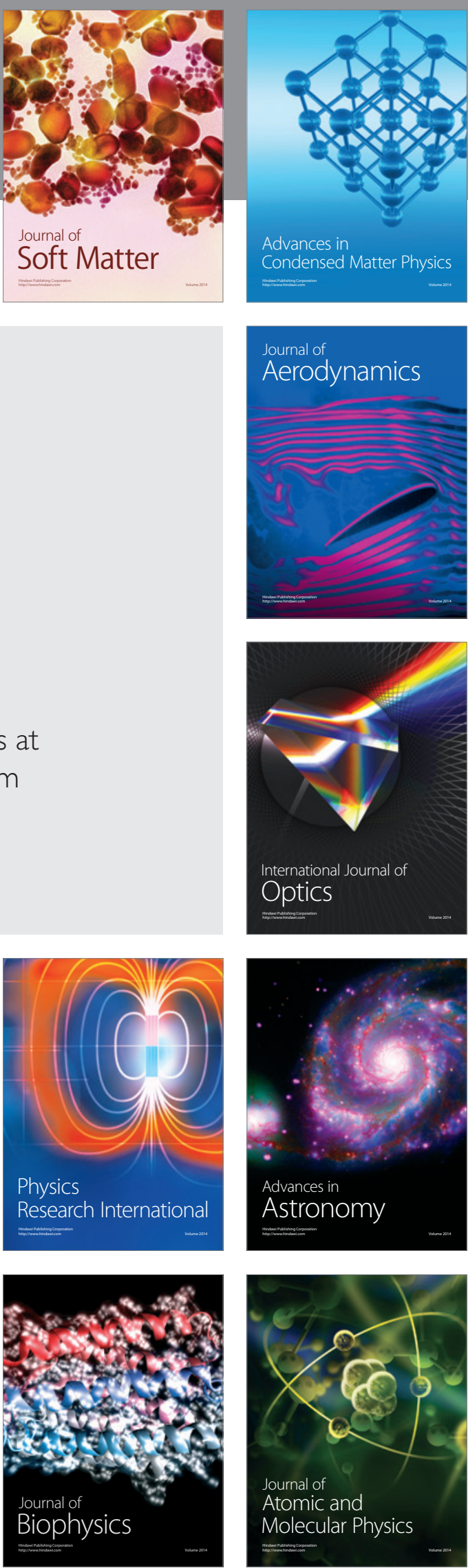